\title{
Work in Progress: A Quantitative Study of Effectiveness in Group Learning
}

\author{
Saket Srivastava and Richa Singh \\ Indraprastha Institute of Information Technology, Delhi \\ New Delhi, India 110078 \\ Email: saket@iiitd.ac.in, rsingh@iiitd.ac.in
}

\begin{abstract}
This work in progress reports an effort towards studying of effect of collaborative effort on student grades. Our motivation is to quantitatively assess the individual and group performance of students in various test settings. This effort would also help the student stimulate interest in group learning and collaboration. Moreover, in addition to stimulating our students interests, we are also augmenting their learning by exposing them towards multiple problem solving approaches while working individually or in groups. This way the students are challenged to use their existing knowledge and approach, and augment it further with the knowledge and approach provided by group partners. Whereas many efforts are focusing on developing new group learning techniques, we intend to study the efficacy of previously proposed techniques under various test settings for $\mathbf{E E}$ and CS courses without significantly diverting from the course framework.
\end{abstract}

\section{INTRODUCTION}

Group learning in students has always been advocated as the best form of knowledge dissemination [1], [2]. The idea seems logical and intuitive as it paves way for collaborative learning environment in which multiple users with different skills and intellect assist each other in learning and understanding a topic. There have been studies in the past that have shown the effectiveness of group learning in certain topics [3], [4]. However, the biggest challenge in evaluating the efficiency of this approach is to establish a testing mechanism that is neither biased nor flawed. It is difficult to quantify the learning progress made by an individual student unless there is an exam that can test a students' ability on a given topic while working individually or in a group. The biggest problem in this quantification is that you give the same set of questions twice, there is a high probability that the second exam using same questions was already compromised since students discussed the answers. At the same time if they are not tested on the same set of questions, there is always a chance that the students may have known one set of questions better than the other. Moreover, if the students already know that they will be evaluated in groups, it may affect their performance as is a possibility that not everyone may contribute to the best of their ability in the group assignment.

Hence the first task towards quantitative evaluation of group learning outcome is to design a testing mechanism that has the least bias and is still technically equivalent in all test cases. Moreover the test should also ascertain the effect of student performance under various marking schemes, multiple choice or single answers with and without negative marking). The rationale behind this approach is that students tend to be more conservative in answering questions when there is a scope of negative marking as compared to non-negative marking. So the testing mechanism we have proposed also takes into account the effect of group learning under such a scheme. We can state that group based performance is better than individual performance only if it holds true under all different types of marking schemes.

In the next section, we start discuss the testing methods employed and some background on the students as well as the subject. Further sections discuss the results and analysis of these testing methods to achieve a quantitative assessment of effectiveness in group learning over individual learning.

\section{BACKGROUND OF TESTING TeChNiques}

We conducted this study in March 2011 and March 2012 with the students of Digital Communication course. This course is offered to second year (fourth semester) students of IIIT Delhi. The students were oblivious to the fact that there will be a surprise quiz as the instructor had only given announced quizzes in the entire semester. Furthermore, the second surprise quiz in the very next class was also a factor in keeping the noise level in this evaluation technique down (by removing the factor of preparation). The students were given a surprise quiz based on the topics already covered in previous classes of the course. The topics covered in this quiz dealt with modulation techniques and bandpass communication techniques.

In the 2011 batch there were 42 students (out of a class size of 57) who took all the quizzes individually and in groups while in the 2012 batch, there were 19 such students (out of a class size of 36) who took all the tests individually as well as in groups. In the first part of this study, we intentionally kept the group size limited to two students. All other students for whom the data was incomplete, were not considered for analysis. No special effort was made to increase class participation in order to maintain the surprise factor.

The first quiz was a mix of 15 multiple choice and fill in the blank type questions and there was no negative marking $(+1$ for correct and 0 for incorrect). There were multiple sets of question papers all having the same questions but different ordering of questions. Once the students completed this quiz, they were asked to repeat the quiz and this time they worked 

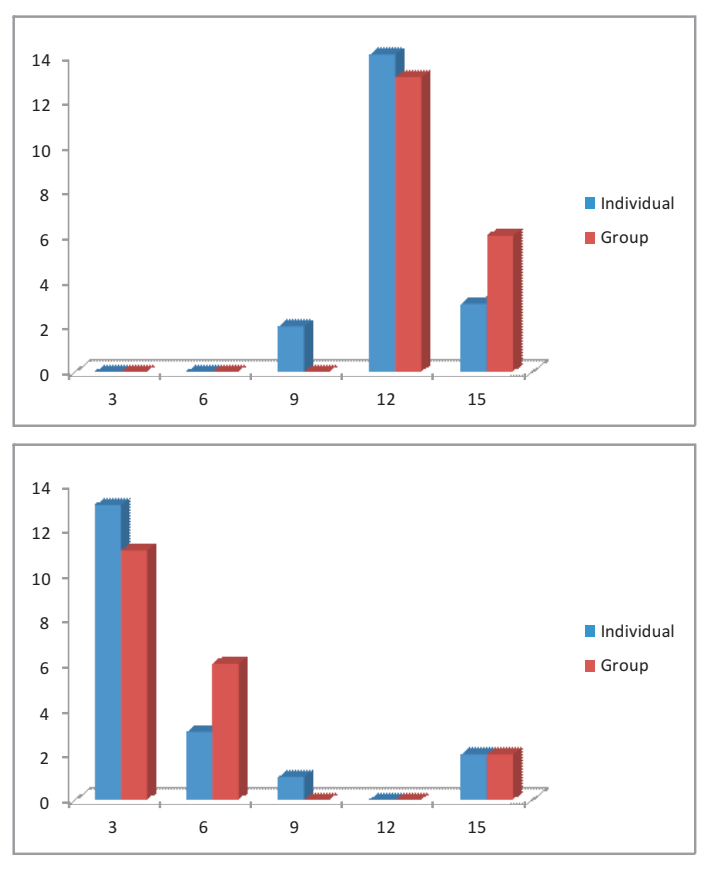

Fig. 1. Histogram demonstrating the performance of 2009 batch students in both quiz 1 (top) and quiz 2 (bottom).

in a group of two students. In the next class, students were given another surprise quiz containing a different set of 20 questions on the same topics covered in quiz 1 . However, this time the quiz had negative marking $(+1$ for correct and -1 for incorrect). Once they finished this quiz, they were again asked to repeat the quiz while working in a group of two students.

\section{ANALYSIS AND RESUlTS}

Since the questions were from similar topics and almost similar in complexity for both quizzes, it was expected that the performance of students, both individually as well as in a group, will be better even if the quiz was negatively marked. The mean and variance for the first quiz, while working alone, was 10.36 and 5.08 respectively and while working in group it was 12.68 and 5.82 respectively. For the second quiz (with negative marking), the mean and variance while working alone was 2.55 and 10.07 respectively and while working in group it was 4.57 and 10.92 respectively.

The histograms in Figures 1 and 2 show that in group quiz, there are more students in the higher bins thus indicating better performance of the students in group quiz than individual quizzes. Analyzing the marks obtained and correlation between group and individual observations shows interesting results. The correlation between the first group and individual study was 0.27 whereas the correlation in the second group and individual quizzes increased to 0.53 . Our results based on rank order test [5] shows that group performance is statistically different and better than individual performance in both the cases.

\section{CONClusion AND Future WORK}

We believe that this work will serve as a good resource for faculties to benchmark the performance of students working
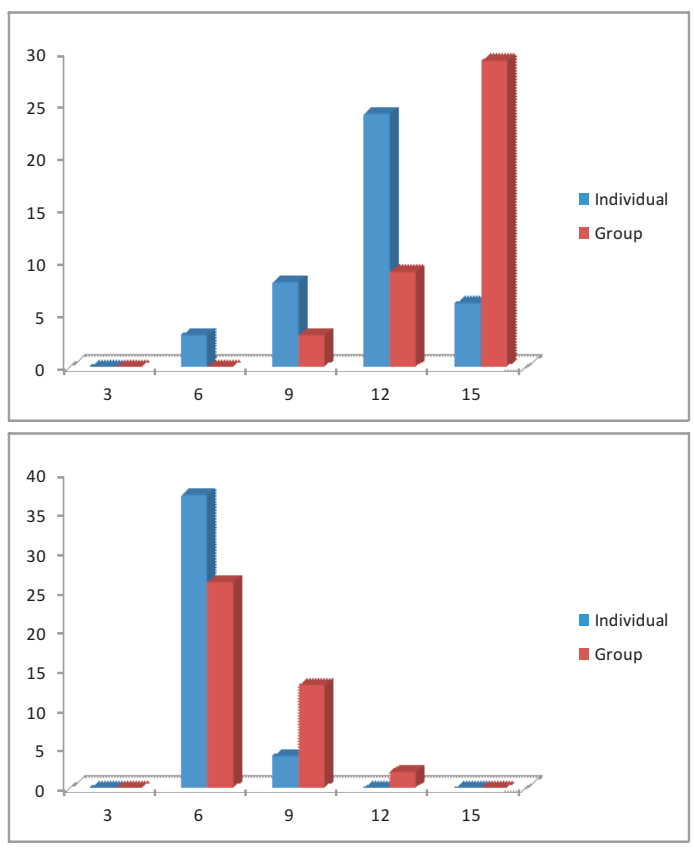

Fig. 2. Histogram demonstrating the performance of 2010 batch students in both quiz 1 (top) and quiz 2 (bottom).

TABLE I

PEARSON CORRELATION BETWEEN INDIVIDUAL AND GROUP QUIZZES.

\begin{tabular}{|c|c|c|}
\hline & $\mathbf{2 0 0 9}$ & $\mathbf{2 0 1 0}$ \\
\hline Quiz 1 Individual - Quiz 1 Group & 0.27 & 0.56 \\
\hline Quiz 2 Individual - Quiz 2 Group & 0.54 & 0.88 \\
\hline Quiz 1 Individual - Quiz 2 Individual & 0.51 & 0.50 \\
\hline Quiz 1 Group - Quiz 2 Group & 0.46 & 0.54 \\
\hline
\end{tabular}

in groups. The deliverables of this work simple quantitative methods that can be used under various grading schemes. In future semesters we also intend to incorporate other kinds of testing schemes and also study the effect of surprise factor on group performance. Another interesting study could be to identify the optimal group size that facilitates maximum learning.

\section{ACKNOWLEDGMENT}

The authors would like to thank 2009 and 2010 batch students of IIIT Delhi, who participated in this study.

\section{REFERENCES}

[1] John Bligh, "Problem based, small group learning," $B M J$, vol. 311, no. 7001, pp. 342-343, 81995.

[2] Leonard Springer, Mary Elizabeth Stanne, and Samuel S. Donovan, "Effects of small-group learning on undergraduates in science, mathematics, engineering, and technology: A meta-analysis," Review of Educational Research, vol. 69, no. 1, pp. 21-51, Spring 1999.

[3] Noreen M. Webb, "Peer interaction and learning in small groups," International Journal of Educational Research, vol. 13, no. 1, pp. 21 - 39, 1989.

[4] K.G. Collier, "Peer-group learning in higher education: The development of higher order skills," Studies in Higher Education, vol. 5, no. 1, pp. 55-62, 1980

[5] M. Hollander and D. A. Wolfe, Nonparametric Statistical Methods, 2nd Edition, Wiley-Interscience, 2 edition, 1999. 